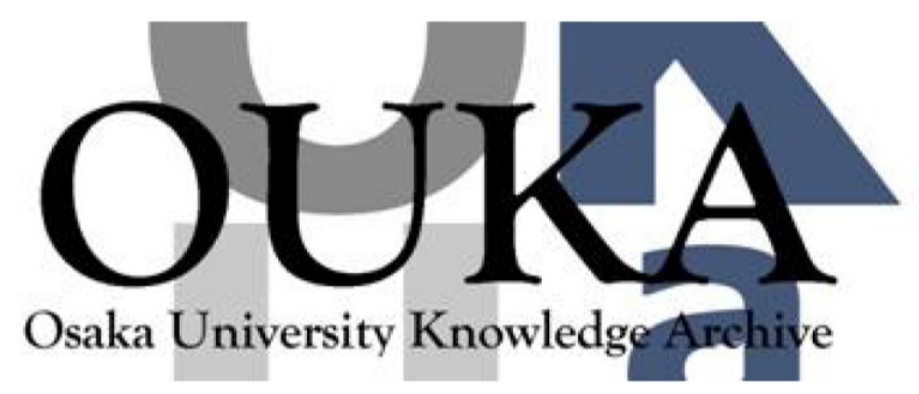

\begin{tabular}{|c|l|}
\hline Title & $\begin{array}{l}\text { Spectrum modulation of relativistic electrons } \\
\text { by laser wakefield }\end{array}$ \\
\hline Author(s) & Nakani i, N.; Kondo, K.; Kuramitsu, Y. et al. \\
\hline Citation & Applied Physics Letters. 93(8) p.081501 \\
\hline Issue Date & 2008-08 \\
\hline oaire:version VoR \\
\hline URL & https://hdl. handle. net/11094/3206 \\
\hline rights & \\
\hline Note & \\
\hline
\end{tabular}

Osaka University Knowledge Archive : OUKA

https://ir. Library. osaka-u. ac. jp/

Osaka University 


\section{Spectrum modulation of relativistic electrons by laser wakefield}

N. Nakanii, ${ }_{1,2, a)}$ K. Kondo, ${ }^{1,2, b)}$ Y. Kuramitsu, ${ }^{2}$ Y. Mori, ${ }^{2,3}$ E. Miura, ${ }^{4}$ K. Tsuji, ${ }^{1,2}$

K. Kimura, ${ }^{1,2}$ S. Fukumochi, ${ }^{1,2}$ M. Kashihara, ${ }^{1,2}$ T. Tanimoto, ${ }^{1,2}$ H. Nakamura, ${ }^{1,2}$

T. Ishikura, ${ }^{1,2}$ K. Takeda, ${ }^{2}$ M. Tampo, ${ }^{2, b)}$ H. Takabe, ${ }^{2}$ R. Kodama, ${ }^{1,2}$ Y. Kitagawa, ${ }^{3}$

K. Mima, ${ }^{1,2}$ and K. A. Tanaka ${ }^{1,2}$

${ }^{1}$ Graduate School of Engineering, Osaka University, 2-1, Yamada-oka, Suita, Osaka 565-0871, Japan

${ }^{2}$ Institute of Laser Engineering, Osaka University, 2-6, Yamada-oka, Suita, Osaka 565-0871, Japan

${ }^{3}$ The Graduate School for the Creation of New Photonics Industries, Hamamatsu, Shizuoka 432-1202, Japan

${ }^{4}$ National Institute of Advanced Industrial Science and Technology, Tsukuba, Ibaraki 305-8568, Japan

(Received 31 August 2007; accepted 28 July 2008; published online 25 August 2008)

Energetic electrons were generated by the interaction of a high-intensity laser pulse with a plasma preformed from a hollow plastic cylinder via laser-driven implosion. The spectra of a comparatively high-density plasma $\sim 10^{19} \mathrm{~cm}^{-3}$ had a bump around $10 \mathrm{MeV}$. Simple numerical calculations explained the spectra obtained in this experiment. This indicates that the plasma tube has sufficient potential to convert a Maxwellian spectrum to a comparatively narrow spectrum. () 2008 American Institute of Physics. [DOI: 10.1063/1.2971235]

Recently, the technology of the ultrashort high-peakpower laser enables us to generate relativistic electrons. These relativistic electrons are of particular importance for the development of laser-accelerators ${ }^{1}$ and fast ignition inertial fusion energy. ${ }^{2}$ In a laser acceleration study, $\mathrm{MeV}$ electrons, which have a Maxwellian spectrum, were generated by the relativistic optical interaction with plasmas using a gas jet. ${ }^{3,4}$ A few years ago, several groups reported the generations of quasimonoenergetic beams, which are essential for a variety of different applications, ${ }^{5}$ by controlling the plasma density and the propagation conditions of the optical pump pulse. ${ }^{6-9}$ More recently, Leemans et al. ${ }^{10}$ succeeded in producing a $1 \mathrm{GeV}$ quasimonoenergetic electron beam using a 3.3-cm-long gas-filled capillary discharge waveguide. In a fast ignition study, Kodama and co-workers ${ }^{11,12}$ demonstrated heating of imploded fuel using a deuteratedpolystyrene shell target with a gold cone. The laser-generated relativistic electrons produced at the tip of the gold cone were used to heat the imploded fuel. In fast ignition studies, it is important not only to increase the coupling efficiency between the optical pulse and the relativistic electrons, but also to control the energy spectrum and the angular distribution of the relativistic electrons, irrespective of whether cone guiding is employed. ${ }^{13,14}$ For example, relativistic electrons with energies under $3 \mathrm{MeV}$ will heat the high-density imploded fuel efficiently. ${ }^{15}$

In this letter, we analyze the energy spectrum of the relativistic electrons produced by GEKKO Petawatt (PW) laser system with a plasma tube, which was generated with six beams of GEKKO XII (GXII) laser system. The spatial density distribution of the plasma tube was controlled by the total energy of GXII laser. This electron beam has an energy spectrum with a bump around $10 \mathrm{MeV}$. The results of a simple numerical analysis indicate that the formation of a bump in the energy spectrum is caused by multidephasing during propagation in the long plasma tube. Thus preformed plasmas within the long plasma tube can act both as an optical guide for the heating laser pulse and as a spectrum

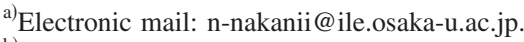

${ }^{b)}$ Present address: Kansai Photon Science Institute, Japan Atomic Energy Agency.
}

modulator for the relativistic electrons. The potentials of these preformed plasmas are considered to be sufficiently high to convert a Maxwellian spectrum to a comparatively narrow spectrum that is effective for heating the fuel in fast ignition.

The experiment was performed using the PW laser system at the Institute of Laser Engineering (ILE) at Osaka University. ${ }^{16}$ As shown in Fig. 1, the preformed plasma tube was created by imploding a hollow polystyrene cylinder that was $3 \mathrm{~mm}$ in length, $700 \mu \mathrm{m}$ in diameter, and had a wall thickness of $14 \mu \mathrm{m}$. To implode this plastic cylinder shell, six beams of the GXII laser system irradiated the outer surface; this irradiation was kept as uniform as possible. The beams for imploding a hollow cylinder were frequency doubled using potassium dihydrogen phosphate crystals, were smoothed by random phase plates, and have the pulse width of $1 \mathrm{~ns}$ in full width at half maximum. The plasma tube, which consists of a central low-density region surrounded by an outer high-density region, is formed during the implosion. The temporal plasma density profile was changed by varying the total energy of the implosion beams $\left(E_{\text {imp }}=1.9,2.0\right.$, and $\left.2.3 \mathrm{~kJ}\right)$. The pump laser beam from the PW laser system was injected $3.1 \mathrm{~ns}$ after the irradiation by the implosion beams. The PW laser system is a chirp pulse amplification Nd:glass laser system having a central wavelength of $1.053 \mu \mathrm{m}$. In the experiment, the pump pulse had an energy of $100 \mathrm{~J}$ and a width of $700 \mathrm{fs}$, with the peak power on target of $140 \mathrm{TW}$. Using f/7.6 optics, the focus

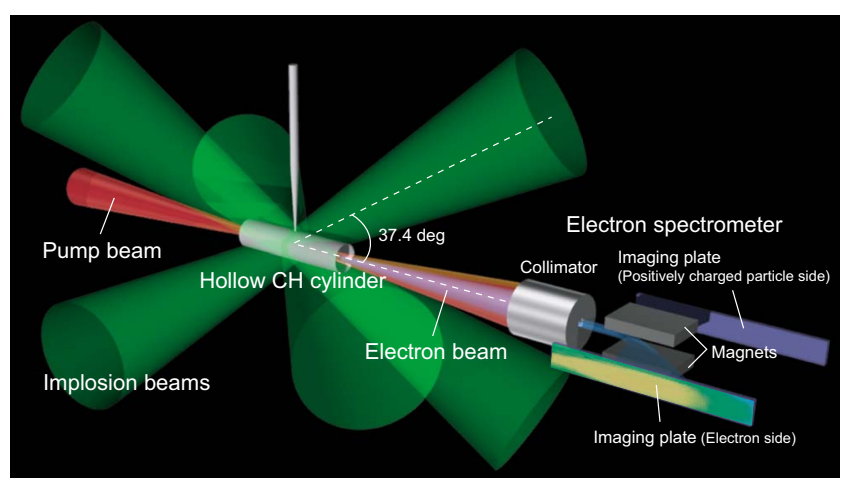

FIG. 1. (Color online) Experimental setup. 


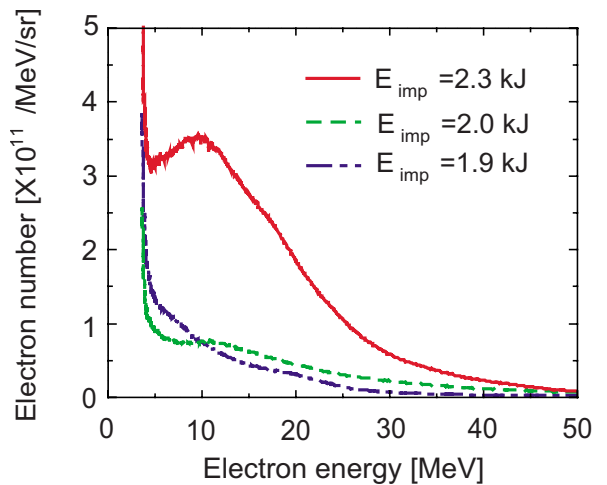

FIG. 2. (Color online) Electron energy spectra plotted with a linear scale for $E_{\text {imp }}=1.9,2.0$, and $2.3 \mathrm{~kJ}$.

diameter of the pump pulse was $65 \mu \mathrm{m}$, which was larger than the diffraction limit of $10 \mu \mathrm{m}$ for $f / 7.6$. Since the pump laser was not perfectly coherent, the length of focused region was roughly estimated to be $7.6 \times 65 \mu \mathrm{m} \sim 500 \mu \mathrm{m}$ from the geometrical condition, but not from the Rayleigh length. This length is much shorter than the length of plasma tube. Then it was really significant to make a plasma tube for a long propagation. The intensity of laser pulse was estimated to be $4.3 \times 10^{18} \mathrm{~W} / \mathrm{cm}^{2}$. The energy spectrum of electrons accelerated forward along the propagation axis of the pump beam was measured by an electron spectrometer (ESM) of which collection solid angle was $2.8 \times 10^{-5}$ sr. An imaging plate (FUJIFILM BAS-SR2025) is used in this ESM as the detector for electrons. ${ }^{17,18}$

Figure 2 shows three spectral profiles of relativistic electrons generated for three different implosion energies with a linear scale. The spectrum for $E_{\mathrm{imp}}=1.9 \mathrm{~kJ}$ has a broad energy spread, which is a characteristic feature of relativistic interactions between the ultraintense laser pulse and plasmas. The electron spectra for $E_{\mathrm{imp}}=2.0$ and $2.3 \mathrm{~kJ}$ have an impressive feature, namely, they have a bump around $10 \mathrm{MeV}$ in the continuous spectrum for comparatively high-density interactions. The highest conversion efficiency from the pump beam to relativistic electrons over $3.8 \mathrm{MeV}$ was calculated to be $4.7 \%$ in this study. This conversion efficiency was estimated by accounting for the angular distribution. The divergence angle of the generated relativistic electron beams were evaluated to be $19^{\circ}$ (half opening angle) by assuming a Gaussian distribution and by using the data from two additional ESMs that were placed in off-axis positions relative to the pump beam's propagation direction. For estimating the average plasma density, we observed the implosion dynamics using x-ray backlight streaked imaging. The density profile of a tentative plasma tube was evaluated by comparing an $\mathrm{x}$-ray backlight streaked image obtained in the experiment with the results of the one-dimensional (1D) hydrodynamics code ILESTA-1D. ${ }^{19}$

We discuss the experimental electron spectra with a simplistic analytic method. The period of the electron plasma wave (EPW) for $\sim 10^{19} \mathrm{~cm}^{-3}$ is much shorter than the pulse width of the pump beam. Thus a self-modulated wakefield could be excited. Under our experimental conditions, the wave breaking can occur, which might be the reason for the electron injection to the laser wakefield. In our model, a simple sinusoidal wakefield with a phase velocity $v_{\mathrm{ph}}=c(1$ $\left.-\omega_{p}^{2} / \omega_{0}^{2}\right)^{1 / 2}$ propagates in the propagation direction of the pump beam. Here $c$ is the speed of light and $\omega_{0}$ is the laser
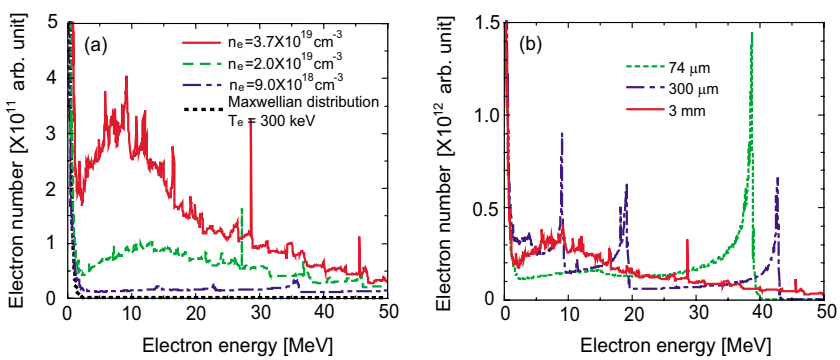

FIG. 3. (Color online) (a) Calculated electron spectra using a simple wakefield acceleration model for three plasma densities, $9.0 \times 10^{18}, 2.0 \times 10^{19}$, and $3.7 \times 10^{19} \mathrm{~cm}^{-3}$, and the spectrum of injected electron beam which has Maxwellian distribution of $300 \mathrm{keV}$. The propagation length is fixed to be $3 \mathrm{~mm}$. (b) Calculated electron spectra for three propagation lengths, $74 \mu \mathrm{m}$, $300 \mu \mathrm{m}$, and $3 \mathrm{~mm}$, respectively. The plasma density is fixed to be 3.7 $\times 10^{19} \mathrm{~cm}^{-3}$.

frequency. The wakefield has the frequency $\omega_{p}$ $=\left(4 \pi n_{e} e^{2} / m\right)^{1 / 2}$, where $n_{e}$ is the plasma density, $e$ is the electron charge, and $m$ is the electron mass. The electric field of the laser wakefield $\left(E_{\text {lwf }}\right)$ is given by

$$
E_{\mathrm{lwf}}(z, t)=E_{\mathrm{wb}} \sin \left(\frac{\omega_{p}}{v_{\mathrm{ph}}} z-\omega_{p} t-\phi\right),
$$

where $\phi$ is the initial phase and $E_{\mathrm{wb}}=m \omega_{p} c / e$ is the wake amplitude in the wave-breaking limit. The wake amplitudes are 280,420 , and $580 \mathrm{GV} / \mathrm{m}$ for $9.0 \times 10^{18}, 2.0 \times 10^{19}$, and $3.7 \times 10^{19} \mathrm{~cm}^{-3}$, respectively. The initial relativistic electrons are assumed to have a Maxwellian energy distribution with the temperature determined by ponderomotive potential energy estimated from the irradiation intensity. These electrons are evenly injected into the first period of the sinusoidal wakefield $(0 \leq \phi<2 \pi)$. Note that we do not consider the energy dependence of the spatial distribution of the injected electrons. However, our model holds the physics of the bump formation qualitatively, as shown below. The detailed model of the injection problem will be discussed elsewhere in the future. We calculate the energy spectrum of the accelerated electrons for a uniform density distribution along the propagation axis. Figure 3(a) shows calculated spectra for three densities of $9.0 \times 10^{18}, 2.0 \times 10^{19}$, and $3.7 \times 10^{19} \mathrm{~cm}^{-3}$, corresponding to $E_{\mathrm{imp}}=1.9,2.0$ and $2.3 \mathrm{~kJ}$ in Fig. 2, respectively. When the plasma density is $3.7 \times 10^{19} \mathrm{~cm}^{-3}$, the spectrum has a bump around $10 \mathrm{MeV}$. Fixing the plasma density to $3.7 \times 10^{19} \mathrm{~cm}^{-3}$, Fig. 3(b) shows the electron spectra for three propagation lengths of $74 \mu \mathrm{m}, 300 \mu \mathrm{m}$, and $3 \mathrm{~mm}$, respectively. The spectrum of $74 \mu \mathrm{m}$ shows the formation of a single quasimonoenergetic spectrum. The spectrum of $300 \mu \mathrm{m}$, multiple quasimonoenergetic peaks are formed. In the spectrum of $3 \mathrm{~mm}$, the isolated quasimonoenergetic peaks are washed out, while the smooth bump structure is formed around $10 \mathrm{MeV}$. These results can be understood as follows: When the electrons have almost the same velocity as the phase velocity of the EPW, they can be trapped and accelerated by the wakefield in a manner similar to the Landau damping. Until the beginning of the deceleration, the accelerated electron spectrum has a quasimonoenergetic structure. Once these electrons have completed the acceleration phase, they enter the deceleration phase and begin to be decelerated. This process is called dephasing. The propagation distance until the commencement of deceleration is referred to as the dephasing length, $\epsilon_{d}=\gamma_{\mathrm{ph}}^{2} \lambda_{p} / 2$, where $\gamma_{\mathrm{ph}}$ $=\left(1-v_{\mathrm{ph}}^{2} / c^{2}\right)^{-1 / 2}$ is the Lorentz factor of the phase velocity of 


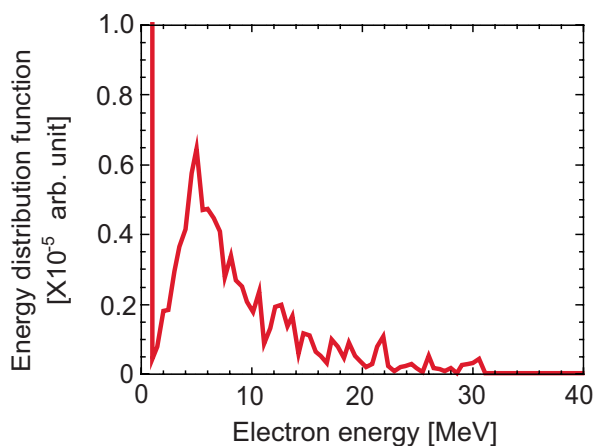

FIG. 4. (Color online) Energy distribution function of electron obtained by 2D-PIC simulation for $n_{e}=3.7 \times 10^{19} \mathrm{~cm}^{-3}$. The plasma length is $1.2 \mathrm{~mm}$.

EPW and $\lambda_{p}=2 \pi v_{\mathrm{ph}} / \omega_{p}$ is the wavelength of EPW. When the plasma density is $3.7 \times 10^{19} \mathrm{~cm}^{-3}$, the dephasing length is estimated to be $74 \mu \mathrm{m}$. When the length of the wakefield plasma is a few times of the dephasing length, multiple quasimonoenergetic peaks are formed in the spectrum, which corresponds to the spectrum of $300 \mu \mathrm{m}$ in Fig. 3(b). When the length of the wakefield plasma is much greater than the dephasing length as the experiments, the trapped electrons are alternately accelerated and decelerated (multidephasing). Eventually, these electrons are concentrated between the energy based on the dephasing length and that based on the phase velocity.

In order to confirm this simple model of electron acceleration, a two dimensional particle-in-cell (2D-PIC) simulation has been performed with OOPIC code. ${ }^{20}$ In this simulation, we use the laser wavelength and peak laser intensity same as the experiments, but with a smaller waist size $w_{0}=20 \mu \mathrm{m}$ and pulse duration $\tau=150$ fs due to our computational restrictions. To model the plasma tube, a density profile is assumed from the result of the 1D hydrodynamics code. The length of this plasma tube is $1.2 \mathrm{~mm}$. Although some of these simulation parameters are different from the experiments, the condition in the simple model can be reproduced. When the central density of plasma tube is 3.7 $\times 10^{19} \mathrm{~cm}^{-3}$, the laser pulse is guided and a self-modulated laser wakefield is excited in the plasma tube. The energy distribution function obtained by 2D-PIC simulation is shown in Fig. 4; the bump structure is formed around $5 \mathrm{MeV}$ in the spectrum. The major candidates why the peak in the PIC is lower than the experimental one are the following: (1) the difference in the self-focusing between $2 \mathrm{D}$ and three dimensional, (2) the ambiguity of the density estimation in the center of the plasma tube from the 1D fluid code, and (3) the density inhomogeneity along the propagation axis of the pump laser in the experiment. However, all the peak values in the experiment, the model, and the PIC are between the phase velocity and the dephasing velocity. Moreover, all of them are close to the phase velocity. The analytic, numerical, and experimental results are qualitatively all consistent.
In conclusion, we have performed laser acceleration experiments using a 3-mm-long plasma tube. The energetic electron spectrum showed a bump around $10 \mathrm{MeV}$. To explain the origin of this bump, we have performed a simple numerical calculation. A bump was obtained at about 10 $\mathrm{MeV}$ in our calculation when the plasma density was 3.7 $\times 10^{19} \mathrm{~cm}^{-3}$. The calculation results indicate that the multidephasing in the EPW causes the formation of this bump during propagation in a long plasma tube. Moreover, we have performed a 2D-PIC simulation and the result reproduced bump structure qualitatively. When the laser and the wakefield can propagate much longer distance than the dephasing length, the bump formation is possible. The governing parameters for the peak velocity are the plasma density and the laser intensity. To keep the laser intensity and, thus, the wakefield amplitude for long distance, the waveguide or, in our case, the density profile is also important to control the process. Our experimental and theoretical results demonstrate that a long plasma tube can act as a spectrum modulator, which converts a Maxwellian spectrum to a comparatively narrow spectrum. An attractive application of this spectrum modulator is using it in a fast ignition study to heat the fuel efficiently.

The authors thank the staff of the TMS, PDT, and GOD groups in ILE for technical support. Moreover, we thank Dr. Sentoku at University of Nevada, Dr. Yabuuchi at UCSD, and Mr. Hama for helpful discussions. This research is supported by grants: (A) type $B$ (Grant No. 18340120) by MEXT, (B) Grant-in Aid for Creative Scientific Research (Grant No. 15GS0214) by MEXT, (C) "High Energy Density Plasma Photonics" by CREST, JST, (D) the Global COE Program, "Center for Electronic Device Innovation," by MEXT (E) the Core-to-Core Program "High Energy Density Science" by JSPS, and (F) type A (Grant No.19206099) by MEXT.

${ }^{1}$ T. Tajima and J. M. Dawson, Phys. Rev. Lett. 43, 267 (1979).

${ }^{2}$ M. Tabak et al., Phys. Plasmas 1, 1626 (1994).

${ }^{3}$ A. Modena et al., Nature (London) 377, 606 (1995).

${ }^{4} \mathrm{~V}$. Malka et al., Science 298, 1596 (2002).

${ }^{5}$ C. Pellegrini and J. Stöhr, Nucl. Instrum. Methods Phys. Res. A 500, 33 (2003).

${ }^{6}$ E. Miura et al., Appl. Phys. Lett. 86, 251501 (2005).

${ }^{7}$ S. P. D. Mangles et al., Nature 431, 535 (2004).

${ }^{8}$ C. G. R. Geddes et al., Nature (London) 431, 538 (2004).

${ }^{9}$ J. Faure et al., Nature (London) 431, 541 (2004).

${ }^{10}$ W. P. Leemans et al., Nat. Phys. 2, 696 (2006).

${ }^{11} \mathrm{R}$. Kodama et al., Nature (London) 412, 798 (2001).

${ }^{12}$ R. Kodama et al., Nature (London) 418, 933 (2002).

${ }^{13}$ Y. Kitagawa et al., Phys. Rev. E 71, 016403 (2005).

${ }^{14}$ K. Fujita et al., Proc. SPIE 4424, 37 (2001).

${ }^{15}$ S. Atzeni, Phys. Plasmas 6, 3316 (1999).

${ }^{16}$ Y. Kitagawa et al., IEEE J. Quantum Electron. 40, 281 (2004).

${ }^{17}$ K. A. Tanaka et al., Rev. Sci. Instrum. 76, 013507 (2005).

${ }^{18}$ N. Nakanii et al., Rev. Sci. Instrum. 79, 066102 (2008).

${ }^{19}$ H. Takabe et al., Phys. Fluids 31, 2884 (1988).

${ }^{20}$ J. P. Verboncoeur et al., Comput. Phys. Commun. 87, 199 (1995). 